\title{
Up-to-date information is in your hands: online teaching
}

\author{
Mustafa Karahan ${ }^{1} \cdot$ Pietro Randelli $^{2}$
}

Received: 21 April 2015 / Accepted: 27 April 2015 / Published online: 14 May 2015

(C) European Society of Sports Traumatology, Knee Surgery, Arthroscopy (ESSKA) 2015

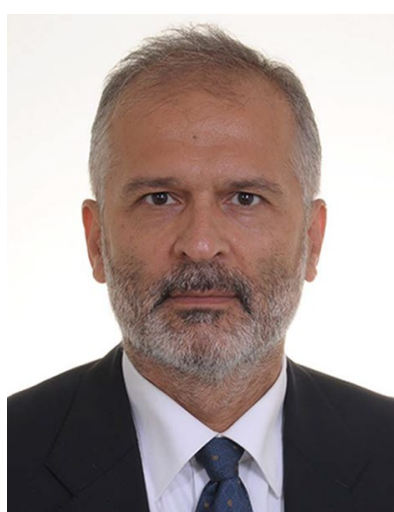

Mustafa Karahan

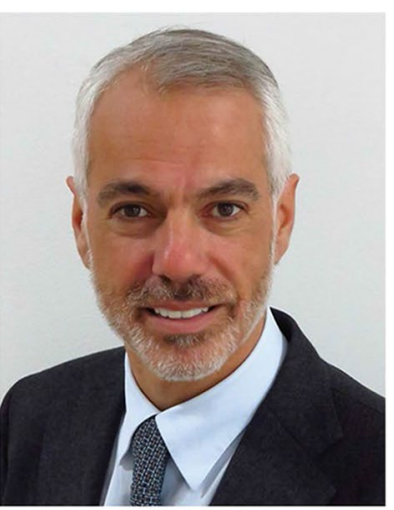

Pietro Randelli
The 'medical profession', which dates back as far as the history of mankind, has seen advances in technology that make their way slowly into the very heart of our daily practice. Innovations that would have amazed us outside our hospital walls are now right in our hands, and we take them for granted. Of all advanced technology available, endoscopic visualisation, magnetic resonance imaging and search engines are the leading ones.

All you need to do to start an arthroscopy procedure to a knee is to push the start button and calibrate the

Mustafa Karahan

drmustafakarahan@gmail.com

Pietro Randelli

pietro.randelli@unimi.it

1 Department of Orthopedics, Acibadem University, Istanbul, Turkey

2 Dipartimento di Scienze Biomediche per la Salute, IRCCS Policlinico San Donato, Università degli Studi di Milano, Via Morandi 30, San Donato Milanese, 20097 Milan, Italy instrument's reception of the colour white to the naked eye's reception of the same colour. The rest is done through a technology so advanced that it made it possible for Apollo 13 to go to the moon and back. To get the colour discrepancies between the damaged and healthy cartilage in MRI, the protons of the tissue are aligned in resting position in a row and followed by a noise recording while they go back to their functioning states. Most of our current readers probably never touched the dusty thick catalogues of the early Index Medicus to look for information, which would take hours if not days, that we can now obtain in minutes, if not seconds, through the current databases on the Internet.

Visual learning is one of the leading styles for surgeons, especially orthopaedic surgeons. Utilisation of advanced technology in the management of our patients' ailments has opened up new frontiers in surgical education and information distribution. Digital recording of patients' status at the diagnostic stage or during surgery has enabled surgeons to present information in a technologically advanced way. Video clips were used in presentations for decades, but recently video presentations have become increasingly popular. As a matter of fact, an audio-visual platform is almost a necessity for an institution aiming to function at an international level.

So, what is the future of surgical training and publication? We are sure that it is going to be more and more digital and online. Online learning reaches the target (the surgeon) through a mobile device with high-quality content which will be probed by the surgeon who will always be curious and bear the need to know more. Currently, smartphones have surpassed the rest as the device to be used while searching for information. However, the future may hold drastic changes such as the new 'Glass systems'. Cloud systems are the preferred data storage systems rather than the institution's own database. The final part of the 
online learning chain is the content available. Information existing on the web is enormous and can be highly contradicting. The liberty that the web provides to the individual opinion holders may lead to subjectivity. It is rare to find a reliable source that is unbiased and commercial free. ESSKA has launched its own Internet educational platform for advanced audio-visual contents and opened it for the membership during the last Congress in Amsterdam.
The site currently holds material coming from ESSKA meetings, documents from the Committees, videos and PowerPoint presentations. Through the ESSKA Academy, members can even access the KSSTA journal and obtain papers they need to be updated about.

We invite all ESSKA members to enter the ESSKA Academy by logging into the site www.esska.org. Let's surf and have fun! 